\title{
Low-dimensional topology, low-dimensional field theory and representation theory
}

\author{
Jürgen Fuchs and Christoph Schweigert*
}

\begin{abstract}
Structures in low-dimensional topology and low-dimensional geometry - often combined with ideas from (quantum) field theory - can explain and inspire concepts in algebra and in representation theory and their categorified versions. We present a personal view on some of these instances which have appeared within the Research Priority Programme SPP 1388 "Representation theory".
\end{abstract}

2010 Mathematics Subject Classification. Primary: 81T45; Secondary: 57R56

Keywords. Topological field theory, tensor categories, categorification

\section{Introduction}

Structures and relations in algebra and representation theory are sometimes "explained" by geometric or topological facts, frequently by facts concerning a category of low-dimensional geometric or topological objects.

As a first illustration, consider the following well-known example of an algebraic relation. Let $A$ be a set. A map $m: A \times A \rightarrow A$ is called associative if for any integer $n \geq 3$ and any given $n$-tuple $\left(a_{1}, a_{2} \ldots, a_{n}\right) \in A^{n}$, every way of putting brackets in the expression $a_{1} a_{2} \cdots a_{n}$ gives, upon applying $m$ repeatedly according to the chosen bracketing, one and the same element of $A$. This is not the typical the textbook definition of associativity; the latter is rather based on the

Proposition 1.1. A map $m: A \times A \rightarrow A$ is associative if and only if the equality

$$
m\left(m\left(a_{1}, a_{2}\right), a_{3}\right)=m\left(a_{1}, m\left(a_{2}, a_{3}\right)\right)
$$

holds for all triples $a_{1}, a_{2}, a_{3} \in A$.

It should be appreciated that this is really an assertion about binary trees with a finite number of leaves. Indeed, any bracketing of $n$ elements corresponds to a binary tree with $n$ leaves. The statement then follows from the observation that any two binary trees can be transformed into each other by applying repeatedly a move that amounts to the associativity relation. In this sense, a combinatorial

\footnotetext{
${ }^{*} \mathrm{CS}$ is partially supported by the Collaborative Research Centre 676 "Particles, Strings and the Early Universe - the Structure of Matter and Space-Time", by the RTG 1670 "Mathematics inspired by String theory and Quantum Field Theory" and by the DFG Priority Programme 1388 "Representation Theory".

JF is supported by VR under project no. 621-2013-4207.

We thank Simon Lentner and Chris Schommer-Pries for helpful comments on the manuscript.
} 
property of the collection of all binary trees "explains" the textbook definition of associativity.

To give an example of a structure "explained" or, rather, justified, by facts from geometry and related to quantum field theory, consider the symmetric monoidal category $\operatorname{Cob}(2,1)$ of smooth oriented cobordisms. The objects of $\operatorname{Cob}(2,1)$ are finite disjoint unions of oriented circles $\mathbb{S}^{1}$, and its morphisms are diffeomorphism classes of smooth oriented surfaces with boundary. One way to learn more about the category $\operatorname{Cob}(2,1)$ is to represent it on a symmetric monoidal category $\mathcal{S}$, i.e. to study symmetric monoidal functors tft: $\operatorname{Cob}(2,1) \rightarrow \mathcal{S}$. (An important example is $\mathcal{S}=$ vect, the category of vector spaces over a field $\mathbb{K}$.) Such a representation tft of the category $\operatorname{Cob}(2,1)$ is called a $(2,1)$-dimensional topological field theory.

For the rest of this section we restrict our attention to $\mathbb{K}$-linear representations, with $\mathbb{K}$ a field, i.e to the case of $\mathcal{S}=$ vect. Representations can be constructed using generators and relations for the object to be represented. The category $\operatorname{Cob}(2,1)$ admits such a description in terms of generators and relations, given by the pairof-pants decomposition of Riemann surfaces with boundary. As a consequence, it suffices to know the vector space

$$
\operatorname{tft}\left(\mathbb{S}^{1}\right)=: C
$$

associated to the circle together with the linear maps $m: C \otimes C \rightarrow C$ and $\Delta: C \rightarrow$ $C \otimes C$ that are obtained from the three-punctured sphere regarded as a cobor$\operatorname{dism} \mathbb{S}^{1} \sqcup \mathbb{S}^{1} \rightarrow \mathbb{S}^{1}$ and as $\mathbb{S}^{1} \rightarrow \mathbb{S}^{1} \sqcup \mathbb{S}^{1}$, respectively, as well as the maps $\eta: \mathbb{K} \rightarrow C$ and $\varepsilon: C \rightarrow \mathbb{K}$ obtained from the disk, seen as a cobordism $\emptyset \rightarrow \mathbb{S}^{1}$ and as $\mathbb{S}^{1} \rightarrow \emptyset$, respectively.

It is well known (see e.g. the textbook 20) that the relations in the pair-ofpants decompositions imply that $(C, m, \Delta, \eta, \epsilon)$ is a commutative Frobenius algebra over $\mathbb{K}$. In this sense, the structure of $\operatorname{Cob}(2,1)$ together with the idea, inspired from quantum field theory, to study representations of $\operatorname{Cob}(2,1)$, enforce on us the algebraic notion of a commutative Frobenius algebra (which was, of course, a known structure long before the advent of topological field theory). The result amounts to a classification of topological field theories, which are objects in the functor category $\operatorname{Fun}_{\otimes, \text { sym }}(\operatorname{Cob}(2,1)$, vect $)$ :

Proposition 1.2. The evaluation on the circle provides an equivalence

$$
\operatorname{Fun}_{\otimes, \mathrm{sym}}(\operatorname{Cob}(2,1), \text { vect }) \simeq \mathrm{Frob}
$$

of categories, where Frob is the groupoid of commutative Frobenius algebras over the field $\mathbb{K}$.

Non-commutative Frobenius algebras and their representation categories can be obtained in a similar spirit when one allows for a larger geometric category $\operatorname{Cob}(2,1)^{\partial}$ that has as objects disjoint unions of circles as well as intervals. One then obtains a so-called open/closed topological field theory. For details, we refer to [21].

In the sequel we will present a small, and strongly biased by personal taste, digest of instances in which the interplay of geometric and algebraic structures has 
lead to a mathematical insight. All those instances are, in some way, related to the Priority Programme "Representation Theory".

\section{Radford's $S^{4}$-theorem and the orthogonal group $\mathrm{SO}(3)$}

Next we present a more recent example for how topological field theory can "explain" a classical fact from algebra. The following statements about a finite-dimensional Hopf algebra $H$ over a field $\mathbb{K}$ can be found in any textbook on Hopf algebras. The Hopf algebra $H$ has a one-dimensional subspace

$$
I_{l}:=\{t \in H \mid h \cdot t=\varepsilon(h) t\}
$$

of left integrals, with $\varepsilon: H \rightarrow \mathbb{K}$ the counit of $H$. By associativity, for $t \in I_{l}$ and any $h \in H$, the element $t \cdot h \in H$ is again a left integral of $H$. Since the space of integrals is one-dimensional, this in turn implies the existence of a linear form $\alpha \in H^{*}$ satisfying $t \cdot h=\alpha(h) t$ for any $t \in I_{l}(H)$. This linear form is a morphism of algebras and thus a group-like element of $H^{*}$. By applying the same reasoning to the dual Hopf algebra $H^{*}$ one finds analogously a distinguished group-like element $a \in H$. A classical result by Radford is

Theorem 2.1 (Radford 30]). Let $H$ be a finite-dimensional Hopf algebra over a field $\mathbb{K}$, and let $a \in H$ and $\alpha \in H^{*}$ be the distinguished group-like elements. Then the fourth power of the antipode $S$ of $H$ satisfies

$$
S^{4}(h)=a\left(\alpha^{-1} \rightarrow h<\alpha\right) a^{-1}=\alpha^{-1} \rightarrow\left(a h a^{-1}\right)\llcorner\alpha
$$

for all $h \in H$.

Here the symbol $\rightarrow$ denotes the left action of $H^{*}$ on $H$ which, using the Sweedler notation $\Delta(h)=h_{(1)} \otimes h_{(2)}$ for the coproduct of $H$, is given by $\alpha \rightarrow h=h_{(1)}\left\langle\alpha, h_{(2)}\right\rangle$, and analogously $<$ is the right $H^{*}$-action on $H$. There is a purely algebraic proof for the purely algebraic statement in Theorem 2.1 .

The statement admits a categorical reformulation. Consider a finite tensor category $\mathcal{C}$, i.e. an abelian rigid tensor category over a field $\mathbb{K}$ in which the monoidal unit is simple and which obeys several finiteness conditions (finite-dimensional morphism spaces, finite Jordan-Hölder length for any object, and finitely many isomorphism classes of simple objects each of which has a projective cover). As an example, take the rigid monoidal category $\mathcal{C}=H$-mod of finite-dimensional modules over a finite-dimensional Hopf algebra $H$. The group-like element $\alpha \in H^{*}$ is a morphism of algebras and thus defines an invertible object $D$ in $\mathcal{C}=H$-mod. Such an invertible object can be introduced for any finite tensor category [12, Lemma 2.9]. Taking into account that the left dual of a module $M=(M, \rho) \in H$ $\bmod$ is given by ${ }^{\vee} M=\left(M^{*}, \rho \circ S^{-1}\right)$ and the right dual of $M$ by $M^{\vee}=\left(M^{*}, \rho \circ S\right)$, Radford's theorem amounts to a relation between the double duals ${ }^{\vee}{ } M$ and $M^{\vee \vee}$ in $\mathcal{C}=H$-mod. Indeed, the assertion in Theorem 2.1 generalizes $[9]$ to the following purely categorical statement: 
Theorem 2.2. Let $\mathcal{C}$ be a finite tensor category. Then there is a natural isomorphism

$$
\vee \vee ? D^{-1} \otimes ?^{\vee \vee} \otimes D
$$

of monoidal functors.

A proof of this statement can be given entirely in a category theoretic setting, see 9 and [32, Sect. 5.2].

We now outline how the theorem can be "understood" in the framework of topological field theories. The definition of a topological field theory as a symmetric monoidal functor can be extended to other cobordism categories, and to highercategorical versions of cobordism categories. In a rather general framework one can consider an $(\infty, n)$-category $\operatorname{Cob}_{\infty, n}^{\mathrm{fr}}$ of $n$-dimensional framed cobordisms, with objects being $n$-framed points, 1-morphisms $n$-framed 1-manifolds with boundary, 2 -morphisms $n$-framed 2-manifolds with corners, etc. For $k>n$, all $k$-morphisms are invertible.

A topological field theory with values in $\mathcal{S}$ is then a symmetric monoidal functor tft: $\mathcal{C}_{\infty} b_{\infty, n}^{\mathrm{f} r} \rightarrow \mathcal{S}$, with $\mathcal{S}$ a symmetric monoidal $(\infty, n)$-category. The same reasoning that shows that the vector space (1) carries additional structure can be used to see that $\mathrm{tft}(*)$, the object assigned to a point, has special properties. The cobordism theorem 23 states that $\operatorname{tft}(*)$ is a fully dualizable object in $\mathcal{S}$ and that any such object determines a framed topological field theory. There is an equivalence

$$
\operatorname{Fun}\left(\operatorname{Cob}_{\infty, n}^{\mathrm{fr}}, \mathcal{S}\right) \simeq k\left[\mathcal{S}^{\mathrm{f} . \mathrm{d} .}\right]
$$

of $\infty$-groupoids generalizing (2), where on the right hand side the symbol $k$ indicates that one discards all non-invertible morphisms in the full subcategory of fully dualizable objects of $\mathcal{S}$.

We now focus on three-dimensional topological field theories. An important target $\mathcal{S}$ is then the symmetric monoidal 3-category $\mathcal{B}$ imod which has finite tensor categories as objects, bimodule categories over finite tensor categories as 1morphisms, and bimodule functors and bimodule natural transformations as 2- and 3-morphisms, respectively. A fusion category, i.e. a semisimple finite tensor category, is a fully dualizable object in $\mathcal{B}$ imod and thus determines a framed three-dimensional topological field theory. Now by change of framing, the orthogonal group $\mathrm{O}(3)$ acts on $\operatorname{Cob}_{\infty, 3}^{\mathrm{fr}}$ and thus on the functor category Fun $\left(\operatorname{Cob}_{\infty, 3}^{\mathrm{fr}}, \mathcal{B}\right.$ imod $)$. This translates into a homotopy action of $\mathrm{O}(3)$ on the fully dualizable objects, i.e. on fusion categories: points in $\mathrm{O}(3)$ give self-equivalences $k\left[\mathcal{B}_{\text {imod }}{ }^{\mathrm{f} . \mathrm{d}}\right.$. $] \rightarrow k\left[\mathcal{B}_{\text {imod }}\right.$ f.d. $]$, paths in $\mathrm{O}(3)$ give natural transformations between self-equivalences, etc. The homotopy groups of the Lie group $\mathrm{O}(3)$ are well-known: The group of connected components is $\pi_{0}(\mathrm{O}(3))=\mathbb{Z}_{2}$; the non-trivial component acts on monoidal categories as $(\mathcal{C}, \otimes) \mapsto\left(\mathcal{C}, \otimes^{\text {opp }}\right)$. The fundamental group is $\pi_{1}(\mathrm{O}(3))=\mathbb{Z}_{2}$; its non-trivial element acts on fusion categories as an autoequivalence, which turns out 8 to be given by the bidual ? ${ }^{\vee \vee}$. Thus from the group-theoretical fact that the nontrivial element of $\pi_{1}(\mathrm{O}(3))=\mathbb{Z}_{2}$ has order 2 one concludes that the quadruple dual ? ${ }^{\vee \vee \vee \vee}$ is trivial, which in turn can be seen to imply Radford's theorem for fusion categories. (Further, there are weakenings on the side of topological field theories 
which allow one to make statements about the quadruple dual of finite tensor categories, see 8.) In this way, topological field theory provides a highly surprising connection between homotopy groups of Lie groups and Radford's $S^{4}$-theorem for finite-dimensional Hopf algebras.

\section{Modularization via equivariant Dijkgraaf-Witten theories}

In the previous section we used topological field theories in three dimensions that are fully extended, i.e. down to the point. In the sequel we study a different variant of three-dimensional topological field theories, which is only extended down to onedimensional manifolds. It is of independent interest for representation theory.

Consider the symmetric monoidal bicategory $\operatorname{Cob}(3,2,1)$ whose objects are closed oriented smooth one-dimensional manifolds, 1-morphisms are oriented surfaces with boundary and 2-morphisms are oriented three-manifolds with corners. To represent this bicategory, consider a symmetric monoidal 2 -functor tft: $\operatorname{Cob}(3,2,1)$ $\rightarrow \mathcal{S}$ with values in a symmetric monoidal bicategory $\mathcal{S}$ : this is a called a 3 -2-1-extended topological field theory. One possible, and particularly important, choice for $\mathcal{S}$ is the symmetric monoidal bicategory 2-vect of so-called 2-vector spaces, which has finite semisimple $\mathbb{K}$-linear abelian categories as objects, $\mathbb{K}$-linear functors as 1-morphisms and natural transformations as 2-morphisms.

In analogy to the situation with $\operatorname{Cob}(2,1)$, the category $\operatorname{tft}\left(\mathbb{S}^{1}\right)$ is endowed with additional algebraic structure, and that structure should determine the three-dimensional topological field theory. Analyzing this structure on $\mathrm{tft}\left(\mathbb{S}^{1}\right)$ with the help of a presentation of $\operatorname{Cob}(3,2,1)$ in terms of generators and relations $[3$, the category $\operatorname{tft}\left(\mathbb{S}^{1}\right)$ turns out to be a modular tensor category, i.e. a braided monoidal semisimple category, together with dualities and a ribbon twist, with non-degenerate braiding. The non-degeneracy condition on the braiding can be formulated in several equivalent ways. A converse statement, that a modular tensor category determines a 3-2-1-extended topological field theory, has already been established long ago 31 .

Topological field theory thus suggests to regard modular tensor categories as a categorified notion of (semisimple) commutative Frobenius algebras. This is indeed a fruitful point of view.

There are several representation-theoretic sources of modular tensor categories:

- Left modules over a connected factorizable ribbon weak Hopf algebra with Haar integral over an algebraically closed field [27.

- Local sectors of a net of von Neumann algebras on $\mathbb{R}$ that has finite $\mu$-index and is strongly additive and split [19].

- Representations of a selfdual $C_{2}$-cofinite vertex algebra with an additional finiteness condition on the homogeneous components and with semisimple representation category [18].

(The last two items constitute different mathematical formalizations of chiral conformal field theories.) Despite these many sources, modular tensor categories are 
"rare" objects 1 As a potential further source of modular tensor categories, crossed modules of finite groups have been proposed [2] some time ago. A crossed module consists of two finite groups $G_{1}$ and $G_{2}$, an action of $G_{2}$ on $G_{1}$ by group automorphisms, and a group homomorphism $\partial: G_{1} \rightarrow G_{2}$ such that

$$
\partial(g . m)=g \cdot \partial(m) \cdot g^{-1} \quad \text { and } \quad(\partial n) \cdot m=n^{-1} \cdot m \cdot n
$$

for all $g \in G_{2}$ and $m, n \in G_{1}$. These data determine a braided monoidal category $\mathcal{C}\left(G_{1}, G_{2}, \partial\right)$ whose objects are $G_{1}$-graded finite-dimensional vector spaces carrying an action of $G_{2}$ such that $g\left(V_{m}\right) \subseteq V_{g . m}$, with the braiding given by

$$
v_{m} \otimes v_{n} \longmapsto \partial(m) \cdot v_{n} \otimes v_{m}
$$

for $v_{n}$ and $v_{m}$ homogeneous elements of degree $n, m \in G_{1}$, respectively. The category $\mathcal{C}\left(G_{1}, G_{2}, \partial\right)$ is a premodular tensor category, i.e. has all the properties of a modular tensor category except that the braiding may be degenerate. It is modular if and only if $\partial$ is an isomorphism, in which case one obtains the representation category of the double of a finite group, i.e. an already known type of modular tensor category. In the general case the category can be modularized according to a standard procedure [5, 25. Somewhat disappointingly, also this modularization turns out to be braided equivalent to the representation category of the Drinfeld double of a finite group.

The modularization leads to a category with a certain weak group action, suggesting that it is actually the neutral part of an equivariant modular category [33. To find equivariant extensions of a given modular tensor category $\mathcal{C}$ is an interesting algebraic problem with deep links to the Brauer-Picard group [11] of $\mathcal{C}$. Again topological field theory, in this case a twisted version of Dijkgraaf-Witten theories, provides one solution to this algebraic problem.

As a simplifying assumption, let us suppose that the group homomorphism $\partial: G_{1} \rightarrow G_{2}$ appearing in the crossed module of finite groups is an injective group homomorphism, so that the group $G_{1}$ is a normal subgroup of $G_{2}$. The corresponding sequence $1 \rightarrow G_{1} \rightarrow G_{2} \stackrel{\pi}{\rightarrow} J \rightarrow 1$ of groups is not necessarily split. But still the group $J$ acts weakly on $G_{1}$, which can be seen as follows. Choose a set-theoretic section $s: J \rightarrow G_{2}$ of $\pi$. Then for any $j \in J$ the automorphism $\rho_{j}\left(g_{1}\right):=s(j) g_{1} s(j)^{-1}$ of $G_{1}$ obeys $\rho_{j_{1}} \circ \rho_{j_{2}}=\operatorname{Ad}_{c_{j_{1}, j_{2}}} \circ \rho_{j_{1} \cdot j_{2}}$ with $c_{i j}:=s(i) \cdot s(j) \cdot s(i \cdot j)^{-1} \in G_{1}$. (We refrain from exhibiting the coherence relations satisfied by these group elements $c_{i j}$.) This weak action induces a (weak) $J$-action on the modularization of the category $\mathcal{C}\left(G_{1}, G_{2}, \partial\right)$ which in the case at hand is the category $\mathcal{D}\left(G_{1}\right)$-mod of modules over the double of $G_{1}$.

For any finite group $G$, the Dijkgraaf-Witten theory provides a 3-2-1-extended topological field theory. The construction is based on the following facts (for a

\footnotetext{
1 This statement should not be interpreted in the sense that modular tensor categories can be classified in a naive way. Indeed, the class of such categories contains the representation categories of all doubles of finite groups, so that a naive classification is impossible. Still, imposing a natural equivalence relation, which amounts to dividing out Drinfeld doubles, one arrives at a Witt group of modular tensor categories [7] which has a strong arithmetic flavor. In applications, this Witt group is a useful recipient of obstructions, see [14].
} 
recent presentation, see 24 and references therein). To any manifold $X$ there is associated the groupoid $\mathcal{A}_{G}(X)$ of $G$-principal bundles. This groupoid is essentially finite if $X$ is compact. (Extended) cobordisms are spans of manifolds, possibly with corners; pull back of bundles yields spans of groupoids. Taking functor categories into the symmetric monoidal category vect amounts to a linearization and results in a 3-2-1-extended topological field theory. In particular one obtains

$$
\operatorname{tft}\left(\mathbb{S}^{1}\right)=\operatorname{Fun}\left(\mathcal{A}_{G}\left(\mathbb{S}^{1}\right), \operatorname{vect}\right) \simeq \mathcal{D}(G) \bmod .
$$

The main idea of the construction of a $J$-equivariant modular category whose neutral component is the modularization of $\mathcal{C}\left(G_{1}, G_{2}, \partial\right)$ is geometric [28]: replace the categories $\mathcal{A}_{G}(X)$ by categories of twisted bundles.

Definition 3.1. Let $J$ act weakly on $G_{1}$, such that there is an exact sequence $1 \rightarrow G_{1} \rightarrow G_{2} \stackrel{\pi}{\rightarrow} J \rightarrow 1$ of finite groups, and let $P \stackrel{J}{\rightarrow} M$ be a $J$-cover over a smooth manifold $M$.

(i) A $P$-twisted $G_{1}$-bundle over $M$ is a pair $(Q, \varphi)$ consisting of a $G_{2}$-bundle $Q$ over $M$ and a smooth map $\varphi: Q \rightarrow P$ over $M$ that obeys

$$
\varphi(q \cdot h)=\varphi(q) \cdot \pi(h)
$$

for all $q \in Q$ and all $h \in G_{2}$.

(ii) A morphism $(Q, \varphi) \rightarrow\left(Q^{\prime}, \varphi^{\prime}\right)$ of P-twisted bundles is a morphism $f: Q \rightarrow Q^{\prime}$ of $G_{2}$-bundles such that $\varphi^{\prime} \circ f=\varphi$.

(iii) The category of $P$-twisted $G_{1}$-bundles is denoted by $\mathcal{A}_{G_{1}}(P \rightarrow M)$.

A $J$-equivariant topological field theory can now be obtained explicitly by a direct generalization of the construction of ordinary Dijkgraaf-Witten theories. In particular, for any group element $j \in J$ there is a $J$-cover $P_{j}$ of $\mathbb{S}^{1}$ with monodromy $j$ and thus a category $\mathcal{C}_{j}=\left[\mathcal{A}_{G}\left(P_{j} \rightarrow \mathbb{S}^{1}\right)\right.$, vect $]$. Then the category $\mathcal{C}:=\bigoplus_{j \in J} \mathcal{C}_{j}$ can be shown 28] to be a $J$-equivariant modular category. Its orbifold category is the category of modules over the Drinfeld double of the group $G_{2}$.

The idea to extend the Dijkgraaf-Witten construction to categories of generalizations of bundles turns out to be quite fruitful. As an illustration consider topological field theories with defects. Such theories are defined on cobordism categories consisting of manifolds with singularities, see e.g. [23, Sect.4.3]. They turn out to be most intimately linked to the theory of module categories over fusion categories 14. Dijkgraaf-Witten theories with defects can be realized using a generalization of relative bundles [15. In this way a combination of field-theoretic and geometric arguments allows one to recover pure algebraic results 29 on module categories over the fusion category of finite-dimensional vector spaces that are graded by a finite group.

\section{Partial dualizations in the theory of Hopf algebras}

The program, initiated by Andruskiewitsch and Schneider, of classifying finitedimensional pointed Hopf algebras over a field has grown into one of the more 
remarkable recent developments in algebra; see 1 for a review. One of the surprises that were encountered in the realization of this program is a close connection between pointed Hopf algebras and Lie theory. The crucial ingredient of this connection is the theory of Weyl groupoids (see [16], as well as [6]).

In this groupoid a construction is central that, while being purely algebraic, turns out to have important ramifications in topological field theory. In the form introduced originally [17, this construction makes extensive use of smash products; a convenient formulation in the framework of braided monoidal categories proceeds as follows.

We first summarize a few facts that are needed in the construction [4]:

- The notions of a Hopf algebra $A$, of a Hopf pairing $\omega: A \otimes B \rightarrow \mathbf{1}_{\mathcal{C}}$, and of the category ${ }_{A}^{A} \mathcal{Y} \mathcal{D}(\mathcal{C})$ of Yetter-Drinfeld modules over $A$ make sense in any braided category $\mathcal{C}$. (Recall that a Yetter-Drinfeld module has the structure of both a module and of a comodule, subject to a certain consistency condition. Yetter-Drinfeld modules form a braided monoidal category.)

- Suppose that we are given two Hopf algebras $A$ and $B$ in $\mathcal{C}$ together with a non-degenerate Hopf pairing $\omega: A \otimes B \rightarrow \mathbf{1}_{\mathcal{C}}$. Using $\omega$ and its inverse, we can turn a left $A$-action into a left $B$-coaction and a left $A$-coaction into a left $B$-action. Hereby we obtain an isomorphism

$$
\Omega^{\omega}: \quad{ }_{A}^{A} \mathcal{Y D}(\mathcal{C}) \stackrel{\cong}{\longrightarrow} \mathcal{Y} \mathcal{D}(\mathcal{C})
$$

of braided categories.

The input of the construction performed in [4] is a partial dualization datum $\mathcal{A}$ for a Hopf algebra $H$ in a braided category $\mathcal{C}$; this consists of a projection $\pi: H \rightarrow A$ to a Hopf subalgebra, and a Hopf algebra $B$ together with a non-degenerate Hopf pairing $\omega: A \otimes B \rightarrow \mathbf{1}_{\mathcal{C}}$. A partial dualization datum $\mathcal{A}$ gives rise to a new Hopf algebra $r_{\mathcal{A}}(H)$ in $\mathcal{C}$ as follows.

(1) The Radford projection theorem, applied to the projection $\pi: H \rightarrow A$, allows us to write the Hopf algebra $H$ as a Radford biproduct $H \cong K \rtimes A$, with $K$ the Hopf algebra in the braided category ${ }_{A}^{A} \mathcal{Y} \mathcal{D}(\mathcal{C})$ given by the coinvariants of $H$ with respect to the projection $\pi$.

(2) The image $L:=\Omega^{\omega}(K)$ of the Hopf algebra $K$ in the braided category ${ }_{A}^{A} \mathcal{Y} \mathcal{D}(\mathcal{C})$ under the braided monoidal equivalence $\Omega^{\omega}$ is a Hopf algebra in the braided category ${ }_{B}^{B} \mathcal{Y} \mathcal{D}(\mathcal{C})$.

(3) The partially dualized Hopf algebra $r_{\mathcal{A}}(H)$ is defined as the Radford biproduct

$$
r_{\mathcal{A}}(H):=L \rtimes B
$$

of $L$. This is a Hopf algebra in the braided category $\mathcal{C}$.

In short, we simultaneously dualize a Hopf subalgebra $A$ of $H$ and transport the coinvariants $K \subseteq H$ covariantly to $L \subseteq r_{\mathcal{A}}(H)$. Note that this combines a contra- 
and a covariant operation; thus partial dualization is not functorial in the Hopf algebra $H$.

From the partial dualization datum $\mathcal{A}$ for $H$ one can obtain a partial dualization datum $\mathcal{A}^{-}$of the Hopf algebra $r_{\mathcal{A}}(H)$. There is then a canonical isomorphism

$$
r_{\mathcal{A}^{-}}\left(r_{\mathcal{A}}(H)\right) \cong H
$$

of Hopf algebras in $\mathcal{C}$, showing that partial dualization is essentially involutive.

The partially dualized Hopf algebra $r_{\mathcal{A}}(H)$ is rather different from $H$, in general. As an illustration consider the Taft algebra $T_{\zeta}$ for some primitive $d$ th root of unity $\zeta . T_{\zeta}$ is a Hopf algebra over the complex numbers that is generated by a group-like element $g$ of order $d$ and a skew-primitive element $x$ having coproduct $\Delta(x)=g \otimes x+x \otimes 1$. There is a projection $\pi$ from the Taft algebra $T_{\zeta}$ to the Hopf subalgebra $A \cong \mathbb{C}\left[\mathbb{Z}_{d}\right]$ generated by $g$. When taking this projection to obtain a partial dualization datum, the resulting partial dualization $r_{\mathcal{A}}(H)$ is in fact isomorphic to the Taft algebra itself, with the isomorphism depending on a choice of a Hopf pairing $\omega: A \otimes A \rightarrow \mathbb{C}$, and thereby on a choice of a primitive $d$ th root of unity. By considering central extensions $\hat{T}_{\zeta, q}$ of $T_{\zeta}$ by group-like elements one can get Hopf algebras with non-trivial partial dualization: the partial dualization $r_{\mathcal{A}}\left(\hat{T}_{\zeta, q}\right)$ no longer contains any central group-like elements. Rather, the coproduct of the skew-primitive element of $\check{T}_{\zeta, q}$ gets modified, in such a way its partial dualization has additional central characters.

A priori the categories of left modules in $\mathcal{C}$ over the Hopf algebra $H$ and over its partial dualization $r_{\mathcal{A}}(H)$ are thus rather different. However, there does exist a representation-theoretic relation between them [4]: The categories of Yetter-Drinfeld modules over a Hopf algebra $H$ in $\mathcal{C}$ and of those over $r_{\mathcal{A}}(H)$ are braided equivalent: we have

$$
{ }_{H}^{H} \mathcal{Y D}(\mathcal{C}) \cong{ }_{K}^{K} \mathcal{Y} \mathcal{D}\left({ }_{A}^{A} \mathcal{Y} \mathcal{D}(\mathcal{C})\right) \stackrel{\Omega^{\omega}}{\longrightarrow}{ }_{L}^{L} \mathcal{Y} \mathcal{D}\left({ }_{B}^{B} \mathcal{Y} \mathcal{D}(\mathcal{C})\right) \cong{ }_{r_{\mathcal{A}}(H)}^{r_{\mathcal{A}}(H)} \mathcal{Y} \mathcal{D}(\mathcal{C})
$$

Here the first and third isomorphisms come from the isomorphism

$$
\underset{K \rtimes A}{K \rtimes A} \mathcal{Y D}(\mathcal{C}) \cong{ }_{K}^{K} \mathcal{Y D}\left({ }_{A}^{A} \mathcal{Y} \mathcal{D}(\mathcal{C})\right)
$$

of braided categories for a biproduct.

The equivalence of categories of Yetter-Drinfeld modules implies a relation between the Hopf algebra $H$ and and its partial dualization $r_{\mathcal{A}}(H)$. Let us discuss this for the case of a finite-dimensional Hopf algebra over a field. The category of Yetter-Drinfeld modules over $H$ is then the Drinfeld center of the category of $H$-modules. Now semisimple algebras having isomorphic centers are Morita equivalent. This statement allows for a categorification: the notion of an algebra is substituted with the one of a monoidal category, while the one of a module over an algebra gets replaced by the one of a module category. This way one arrives 10 , Thm 3.1] at the statement that semisimple tensor categories with braided-equivalent Drinfeld centers have equivalent bicategories of module categories, i.e. they are related by what is called [26] a weak monoidal Morita equivalence. It is natural 
to ask whether the bicategories of module categories over $H$-mod and over $r_{\mathcal{A}}(H)$ mod are closely related, possibly even equivalent. This in turn suggests that the categorified representation theory of a Hopf algebra $H$, i.e. the theory of module and bimodule categories over the monoidal category $H$-mod, should be regarded as an important aspect of the theory of Hopf algebras.

Since the Brauer-Picard group of the monoidal category $H$-mod is isomorphic to the group of braided equivalences of the Drinfeld center $\mathcal{Z}(H$-mod) [11, it is invariant under partial dualization. On the other hand it is not hard to give conditions 22 that ensure the existence of a Hopf isomorphism $f: r_{\mathcal{A}}(H) \stackrel{\cong}{\rightrightarrows} H$. (There is also a generalization involving a modified partial dualization of $H$.) Every pair $\left(r_{\mathcal{A}}, f\right)$ then induces an equivalence

$$
\mathcal{Z}(H \text {-mod }) \simeq \mathcal{Z}\left(r_{\mathcal{A}}(H) \text {-mod }\right) \simeq_{f} \mathcal{Z}(H \text {-mod })
$$

and thus provides an element in the group of braided autoequivalences of the Drinfeld center $\mathcal{Z}(H$-mod), and thereby an element of the Brauer-Picard group.

This result has an interesting application in three-dimensional topological field theories of Turaev-Viro type. A Turaev-Viro theory is a fully extended oriented three-dimensional topological field theory, which assigns a spherical fusion category $\mathcal{A}$ to the point. Types of topological surface defects in this theory are given by $\mathcal{A}$-bimodule categories. Now it is a general field-theoretic pattern that topological invertible codimension-one defects are related to symmetries. As a consequence, the Brauer-Picard groups of the fusion categories in question can be identified [13. as symmetry groups of three-dimensional topological field theories of Turaev-Viro type. In the special case that the fusion category is the category of modules over the group algebra $H=\mathbb{C}[G]$ of an abelian group $G$, the reflections just discussed amount to what in the physics literature are known as electric-magnetic dualities. This constitutes yet another instance of an enlightening relation between algebraic notions and notions from field theory. 


\section{References}

[1] N. Andruskiewitsch, On finite-dimensional Hopf algebras. Proceedings of the ICM 2014, S.Y. Jang, Y.R. Kim, D.W. Lee, and I. Yie, eds. Kyung Moon, Seoul, Vol. II, pp. 117-141 (2014). arXiv:1403.7838 [math.QA].

[2] P. Bantay, Characters of crossed modules and premodular categories. In: Moonshine: The First Quarter Century and Beyond, J. Lepowsky, J. McKay, and M. Tuite, eds. Cambridge University Press, Cambridge, 2010. arXiv:math/0512542 [math.QA].

[3] B. Bartlett, C. Douglas, C. Schommer-Pries, and J. Vicary, Modular categories as representations of the 3-dimensional bordism 2-category. Preprint arXiv:1509.06811 [math.AT].

[4] A. Barvels, S. Lentner, and C. Schweigert, Partially dualized Hopf algebras have equivalent Yetter-Drinfel'd modules. J. Algebra 430 (2015) 303-342. arXiv: 1402.2214 [math.QA].

[5] A. Bruguières, Catégories prémodulaires, modularisations et invariants des variétés de dimension 3. Math. Annalen 317 (2000) 215-236.

[6] M. Cuntz and S. Lentner, A simplicial complex of Nichols algebras. Preprint arXiv: 1503.08117 [math.QA].

[7] A. Davydov, M. Müger, D. Nikshych, and V. Ostrik, The Witt group of nondegenerate braided fusion categories. J. reine angew. Math. 677 (2013) 135-177. arXiv:1009.2117 [math.QA].

[8] C. Douglas, C. Schommer-Pries, and N. Snyder, Dualizable tensor categories. Preprint arXiv:1312.7188 [math.QA].

[9] P. Etingof, D. Nikshych, and V. Ostrik, An analogue of Radford's $S^{4}$ formula for finite tensor categories. Int. Math. Res. Notices 54 (2004) 2915-2933. arXiv:math/0404504 [math.QA].

[10] P. Etingof, D. Nikshych and V. Ostrik, Weakly group-theoretical and solvable fusion categories. Adv. Math. 226 (2011) 176-205. arXiv:0809.3031 [math.QA].

[11] P. Etingof, D. Nikshych, V. Ostrik, and E. Meir, Fusion categories and homotopy theory. Quantum Topology 1 (2010) 209-273, arXiv:0909.3140 [math.QA].

[12] P. Etingof and V. Ostrik, Finite tensor categories. Moscow Math. J. 4 (2004) 627-654. arXiv:math/0301027 [math.QA].

[13] J. Fuchs, J. Priel, C. Schweigert and A. Valentino, On the Brauer groups of symmetries of abelian Dijkgraaf-Witten theories. Commun. Math. Phys. 339 (2015) 385-405. arXiv:1404.6646 [hep-th].

[14] J. Fuchs, C. Schweigert and A. Valentino, Bicategories for boundary conditions and for surface defects in 3-d TFT. Commun. Math. Phys. 321 (2013) 543-575, arXiv: 1203.4568 [hep-th].

[15] J. Fuchs, C. Schweigert and A. Valentino, A geometric approach to boundaries and surface defects in Dijkgraaf-Witten theories. Commun. Math. Phys. 332 (2014) 9811015. arXiv:1307.3632 [hep-th].

[16] I. Heckenberger, The Weyl groupoid of a Nichols algebra of diagonal type. Inv. Math. 164 (2006) 175-188. arXiv:math/0411477 [math.QA].

[17] I. Heckenberger and H.-J. Schneider. Adv. Math. 144 (2013) 354-394. arXiv: 1111.4673 [math.QA] 
[18] Y.-Z. Huang, Vertex operator algebras, the Verlinde conjecture and modular tensor categories. Proc. Nat. Acad. Sc. 102 (2005) 5352-5356. arXiv:math/0412261 [math.QA].

[19] Y. Kawahigashi, R. Longo, and M. Müger, Multi-interval subfactors and modularity of representations in conformal field theory. Commun. Math. Phys. 219 (2001) 631669. arXiv:math/9903104 [math.QA].

[20] J. Kock, Frobenius Algebras and 2D Topological Quantum Field Theories. London Mathematical Society Student Texts 59, Cambridge University Press, Cambridge, 2003.

[21] A. Lauda and H. Pfeiffer, Open-closed strings: two-dimensional extended TQFTs and Frobenius algebras. Topology and its Applications 155 (2008) 623-666, arXiv: math/0510664 [math.AT].

[22] S. Lentner and J. Priel, A decomposition of the Brauer-Picard group of the representation category of a finite group. Preprint arXiv:1506.07832 [math.QA].

[23] J. Lurie, On the classification of topological field theories. Current Developments in Mathematics 2008 (2009) 129-280. arXiv:0905.0465 [math.CT].

[24] J.C. Morton, Cohomological twisting of 2-linearization and extended TQFT. J. Homotopy and Related Structures 10 (2015) 127-187. arXiv:math/1003.5603 [math.QA].

[25] M. Müger, Galois theory for braided tensor categories and the modular closure. Adv. Math. 150 (2000) 151-201. arXiv:math/9812040 [math.CT].

[26] M. Müger, From subfactors to categories and topology I. Frobenius algebras in and Morita equivalence of tensor categories. J. Pure Appl. Algebra 180 (2003) 81-157. arXiv: math/0111204 [math.CT].

[27] D. Nikshych, V. Turaev, and L. Vainerman, Quantum groupoids and invariants of knots and 3-manifolds. Topology and its Appl. 127 (2003) 91-123. arXiv: math/0006078 [math.QA].

[28] J. Maier, Th. Nikolaus and C. Schweigert, Equivariant modular categories via Dijkgraaf-Witten theory. Adv. Th. Math. Phys. 16 (2012). arXiv:1103.2963 [math.QA].

[29] V. Ostrik, Module categories over the Drinfeld double of a finite group. Int. Math. Res. Notices 27 (2003) 1507-1520. arXiv:math/0202130 [math.QA].

[30] D.E. Radford, The order of the antipode of a finite dimensional Hopf algebra is finite. Amer. J. Math. 98 (1976) 547-597.

[31] N.Yu. Reshetikhin and V.G. Turaev, Invariants of 3-manifolds via link polynomials and quantum groups. Inv. Math. 103 (1991) 547-598.

[32] K. Shimizu, On unimodular finite tensor categories. Preprint arXiv:1402.3482 [math.QA].

[33] V.G. Turaev, Homotopy Quantum Field Theory. EMS Tracts in Mathematics Vol. 10, European Mathematical Society, Zürich, 2010.

Algebra und Zahlentheorie, Fachbereich Mathematik, Universität Hamburg, Bundesstraße 55, 20148 Hamburg, Germany

E-mail: Christoph.Schweigert@uni-hamburg.de

Teoretisk fysik, Karlstads universitet, Universitetsgatan 21, 65188 Karlstad, Sweden

E-mail: juergen.fuchs@kau.se 